\title{
The Effect of Implementing an SMS Messaging System to Overcome the Lack of Transmission of HPV Facts in Saudi Arabia
}

\author{
Hind Bitar \\ King Abdulaziz University \\ hbitar@kau.edu.sa
}

\author{
Terry Ryan \\ Claremont Graduate University \\ Terry.ryan@cgu.edu
}

\author{
Sarah Alismail \\ Claremont Graduate University \\ sarah.alismail@.cgu.edu
}

\begin{abstract}
Human papillomavirus (HPV) infections are the leading cause of cervical cancer, which kills hundreds of thousands of women around the world. Facts about $H P V$ exist, yet there is a lack of knowledge about it among women who need to know. One factor that limits the spread of knowledge is that HPV is marked by stigma. Another factor is language barriers. The main aim of this research is to develop and implement a "theory of the solution" (Markus, 2014). The solution designed involves a 2-way interactive messaging system, directed by an HPVWA flowchart, to increase and maintain women's self-efficacy to educate themselves about HPV. The system is one possible solution to the problem of the lack of transmission of HPV facts. 34 Saudi women participated in the research. Its results indicate that using an SMS messaging system is an effective method to use to increase and maintain women's self-efficacy.
\end{abstract}

\section{Introduction}

HPV is a sexually transmitted disease (STD), common among sexually-active adults [1]. Wong and Sam report that $30-60 \%$ of sexually-active people get infected with HPV, women of college-age being especially at risk. According to Walboomers et al. (1999), HPV is the leading cause of cervical cancer, with an HPV infection preceding the cancer diagnosis in an estimated $99.7 \%$ of all cases [2]. Cervical cancer is one of the leading causes of death of women around the world [3]. The World Health Organization (WHO) estimated 570,000 new cervical cancer cases in 2018, with around $90 \%$ of deaths happening in low- and middle-income countries [4].

Knowledge about HPV exists in many public information sources--e.g., the "Mayo Clinic" website-but unfortunately, this knowledge does not necessarily reach women who must know about this sexuallyrelated illness [5]. This infection is marked with stigma, which many see as a reason for lacking knowledge about it [5]. STDs, including HPV, are linked to stigma, and this link affects individuals seeking sexual health information to increase their general knowledge, to avoid STDs. Some Saudi women reported that this stigma leads them to avoid discussing HPV with their close family members [6]. Also, from a cultural perspective, single women are less likely to discuss HPV compared to married women [6]. Another factor that affects information seeking among Arab women is a lack of Arabic materials--a language barrier. Arab women seem to stop their attempts to search for information about STDs if they cannot find information in Arabic to read, hear, or watch. Furthermore, searching for and learning from health materials written in different languages affects women's seeking sexual health information to increase their knowledge [6]. Thus, the problem of failure of transmission of HPV facts must be addressed by society, including the IS/IT research community [5].

As a start to addressing this problem, this research develops a type of theory proposed by Markus, a theory of the solution [7]. This type of theory focuses on designing and building an IT artifact as a solution to the problem that was developed previously as a theory of the problem [7] - in this case, lack of transmission of HPV facts [5]. This research follows up research that was conducted by Bitar and Ryan [5] and Bitar et al. [6]. The authors in these previous studies established a theory of the problem through developing and understanding the eight factors in the HPV facts transmission model [5] that explain the lack of transmission of the HPV facts among women in Saudi Arabia. Based on the HPV facts transmission model, there are several mechanisms involved in the transfer of HPV facts to increase women's knowledge, such as having a good intention and coping planning [6]. This research has only one objective--to increase and maintain women's self-efficacy to educate themselves regarding HPV to increase their knowledge of it. This research asks and answers only one question: how can IS/IT enhance and maintain women's self-efficacy to educate themselves about HPV? The researchers 
developed and supported a theory of the solution by creating and testing a 2-way interactive messaging system that implemented the HPV Women's Awareness (HPVWA) flowchart. This solution has the potential to play a significant role in alleviating the lack of transmission of HPV facts. The HPVWA flowchart describes the steps followed in sending SMS messages to the participants to enhance and maintain their selfefficacy to educate themselves about HPV.

Together, these two theories with the implementation of the HPVWA flowchart into the 2way interactive messaging system allow us to pursue improvement in the current state of affairs regarding what Saudi women at risk know about HPV and how to avoid it. It is to be hoped that this will be a significant, albeit small, step towards keeping as many women as possible from contracting cervical cancer.

\section{Background Literature}

\subsection{HPV and cervical cancer}

There is a well-established association between HPV and cervical cancer [8]. Cervical cancer is a significant public health problem, being both the most common cancer type among women and the one that causes the most deaths [3,9]. With early diagnosis, cervical cancer is preventable, and may even be curable. However, most Saudi women typically are diagnosed or seek treatment when in the advanced stages, which consequently reduces the rate of survival [10]. A hospital-based study of a cohort of mostly Saudi women reported a high prevalence of $\mathrm{HPV}$, with $17 \%$ of 400 participants testing positive for HPV [10]. Additionally, in Saudi Arabia, there is no HPV immunization program in which HPV vaccines are available by patient request [11].

There are many varieties of HPV infection, but HPV types 16 and 18 are the cause of more than two-thirds of all cervical cancer cases [3]. It has been reported that the rate of cervical cancer is higher in less-developed countries compared to more-developed countries [1]. Women diagnosed with cervical cancer are usually aged 35 to 50; it infrequently affects women aged 20 or younger. Around $20 \%$ of women diagnosed with cervical cancer are aged 65 or older [12].

\subsection{HPV lack of knowledge and Arabic material}

Several studies have shown that there is a lack of knowledge about HPV infections among women around the world. For example, women in Western countries have very little knowledge about HPV infections, even highly-educated ones [1]. Also, according to Al-Shaikh et al., around $95 \%$ of Saudi female students in health colleges had poor knowledge about this sexual infection [13]. Another study had been conducting in three different areas in Birmingham to identify knowledge and attitudes regarding HPV vaccination. A total of 420 participants between 16 and 54 years old were recruited. According to the authors, $81 \%$ had a zero score of knowledge regarding HPV infection and its link to cervical cancer [14]. Similar results have provided by Wong, and according to the author, women in Rural Southeast Asia country have very little knowledge about HPV and cervical cancer risks. As remarked in many studies, providing HPV education programs to women could help to increase their knowledge about HPV $[1,15]$.

Even though there are many websites, research papers, blogs, and other outlets that discuss and explain many diseases, including symptoms and preventions, there remains a dearth of knowledge about these diseases. One reason is the lack of Arabic health materials. Bitar and Ryan propose eleven propositions concerning the transmission of HPV facts to women who could benefit from them; one of these is language barriers [5]. The authors defined this proposition as "U.S residents who speak little English face several health care issues because of Language Barriers. Talking with someone who speaks another language and/or reading health materials written in another language will impede HPV Facts Transmission" [5:4218]. In 2019, Bitar et al. conducted follow-up research, a qualitative case study to better understand the phenomenon by theorizing about the lack of transmission of HPV facts and lack of awareness of HPV infection among Saudi women. The results show that language barriers serve as a factor that can block the transmission of HPV facts [6]. As reported by some of the participants (Saudi women), if there were no Arabic materials available, they would stop their searching and learning about HPV [6]. One participant reported that "I search and learn using the Arabic Language even though there are not a lot of materials in Arabic." Shah et al. declare that a language barrier is among the most significant obstacles to healthcare access. One participant questions "why more Arabic resources were not available" (p.432) [16]. Lack of Arabic materials is one reason for Saudi women having insufficient knowledge of HPV infections.

\subsection{Theoretical foundation: HPV facts transmission model, including barriers and resources factors}

As stated before, this research study is follow-up research to earlier work by Bitar and Ryan [5] and Bitar 
et al. [6]. Bitar and Ryan theorized about the problem of lack of transmission of HPV facts by proposing a conceptual model, HPV Facts Transmission Model, including barriers and resources factors. This model was developed based on the Health Action Process Approach (HAPA) Model, as shown in Figure 1. The conceptual model has 11 propositions that may promote or block the transmission of HPV facts. These propositions were divided into two primary levels, which are individual and social.

In 2019, Bitar et al. conducted follow-up research, which is a qualitative case study in Saudi Arabia to understand the lack of knowledge/awareness about HPV infection among women who could benefit from existing HPV facts [6]. The authors used a pattern matching to analyze the data. As a result, there are eight of the eleven propositions that affect the transmission of HPV facts in Saudi Arabia case study, as shown in Figure 2. The authors divided these factors into barriers and resources [6].

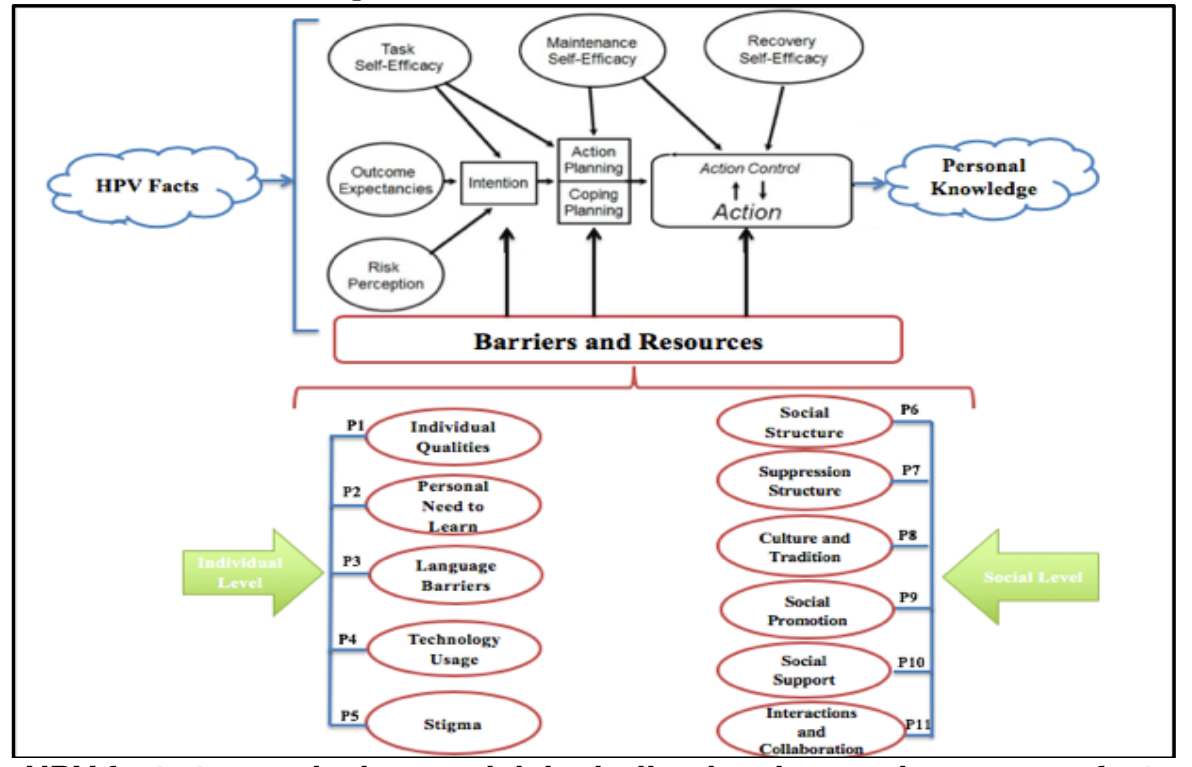

Figure 1. HPV facts transmission model, including barriers and resources factors [5]

The understanding of these factors in the HPV facts transmission model explains the lack of transmission of the HPV facts, which establish a theory of the problem. This theory stated that HPV facts exist in many places, such as background literature and education programs. Women can start searching to increase their knowledge about HPV by reading, listening, talking, and watching any of the available sexually health materials. During the searching time, women could discover the link between HPV and cervical cancer. These women realize that if they do not try to increase their knowledge, they will be more likely to contract HPV and possibly develop cervical cancer, which is a risk perception (threats) in the HPV facts transmission model. On the same time, women may start to think positively about the consequences of health behavior. For example, "if I search and learn more regarding the HPV infections, I may reduce the chance of being infected, so I will not have a cervical cancer," this reflected the outcome expectancies as shown in the model (see Figure 2). Having a balance between these two predictors and having high task self-efficacy help women to establish and maintain a firm intention to continue searching and learning about HPV. Such a balance is reflective of the motivation phase $[5,6]$.

Moving to the volition phase, one can observe several mechanisms that could assist women in increasing their knowledge about HPV. The first of these is action planning. Having a good intention helps women to start their action planning by concentrating on when, where, and how to perform a new action, in this case searching and learning about HPV. The second mechanism is coping planning. Here, women may imagine a scenario where and when they cannot perform the searching and learning about HPV to increase their knowledge [5,6]. For example, "If I want to search and learn about HPV, but I am afraid of not finding the Arabic materials to read and learn, I will not stop, I will ask my doctors about any information that I want to learn." According to Sniehotta, Scholz, and Schwarzer, coping planning focuses on the unintended responses, such as habits and intentional responses, such as newly planned actions [17]. Action planning is considered as an essential step to begin a health behavior change, while coping planning is vital to begin and maintain the searching and learning about HPV [17]. 


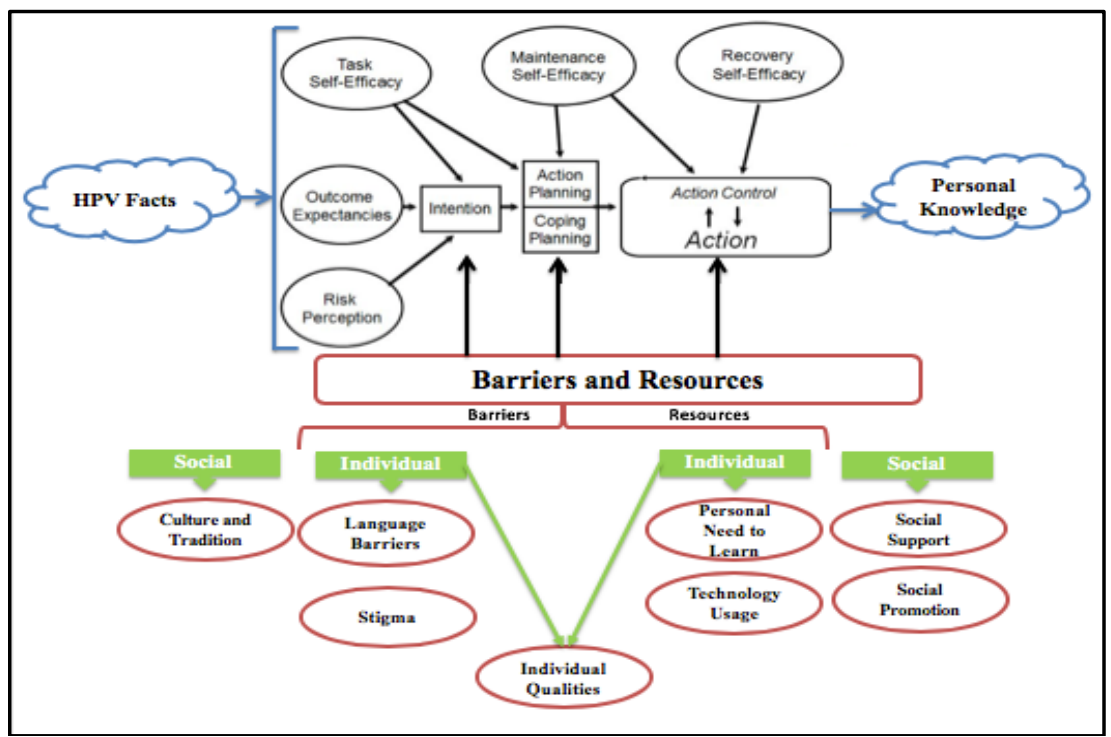

Figure 2. HPV facts transmission model, including barriers and resources after analysis [6]

After these two steps, action planning and coping planning, women will continue their searching and learning actions with action control until they get the personal knowledge that they need $[5,6]$. In the second phase and during these mechanisms, there are essential predictors to continue searching and learning about $\mathrm{HPV}$, which are maintenance self-efficacy and recovery self-efficacy. These women surrounded by people and live in a society, so there is a considerable impact from them-this reflects the barriers and resources component in the model. The barriers and resources are divided into two main sub-levels, which are individual and social level $[5,6]$. Under each sub-level, there are one, two, or three factors that affect the transmission of HPV facts, as shown in Figure 2. The individual-level factors are personal need to learn, technology usage, language barriers, stigma, and individual qualities; the social-level factors are social promotion, social support, and culture and tradition [6]. This model was used as a theoretical foundation to develop the IT solution - the two ways interactive SMS messaging system.

\section{Designing the IT solution}

To address the objective of this current research and since there are several mechanisms involved in HPV facts transmission (see Figure 2), the research scope is the motivation phase and the two steps of the volition phase, which are action planning and coping planning. Therefore, the IT artifact was designed and used to increase and maintain task self-efficacy to educate Saudi women about HPV infections (see Figure 3).

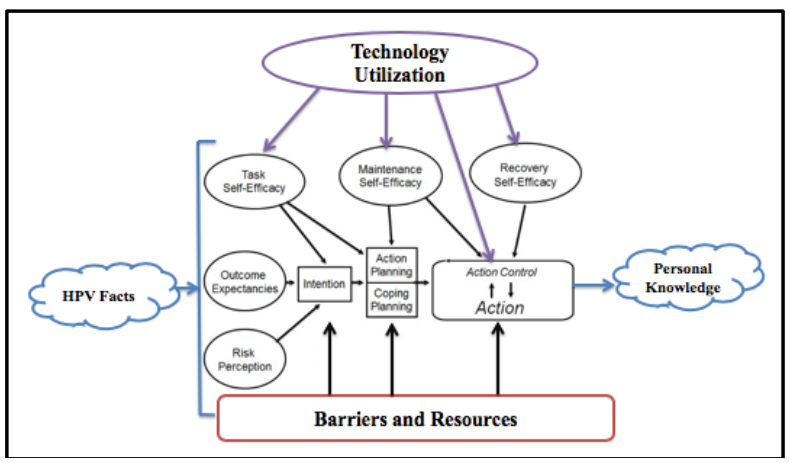

Figure 3. Technology utilization in HPV facts transmission model $[5,6]$

\subsection{Identifying the IT artifact}

Bitar et al. went through three different stages to identify the IT artifact that could aid in transferring the HPV facts among Saudi women. In the first stage, the authors asked the participants a few questions about the type of technology that they would use to educate themselves about the stigmatized HPV. The participants suggested three types of IT artifact, which are simple text messages through WhatsApp, a simple-to-use educational mobile application, or a simple webpage that include any critical information that must be known about the HPV infection [6].

Then, to narrow down the suggestions, the authors moved to the next stage, and they developed a short survey and distributed it to Saudi women using WhatsApp [6]. The authors received 966 responses, and they suggest the following: 
1. Around half of the participants want to learn and increase their knowledge about any STDs using simple technology, such as text messaging.

2. About $37.8 \%$ of the participants suggest to download and use an educational app that contains information about STDs, such as HPV.

The most important suggestion by the participants (with a high percentage, around $95.4 \%$ ) is that they prefer to learn and educate themselves about these diseases using Arabic [6].

The suggestions narrow down to two, so the authors moved to the third and last stage to identify the IT artifact that could be used by Saudi women to educate themselves about HPV. The first author developed a second survey guided by stage 2 responses and distributed it to Saudi women using WhatsApp. The reason behind developing this second survey was to choose between these two IT artifacts and to understand the reasons behind selecting a specific type of technology. The results of this survey showed that $60 \%$ of the participants prefer to receive simple text messages regularly [6]. Their reasons were as follow:

1. Receiving simple text messages are suitable for all ages.

2. This technology will force women to read and learn without the need of having strong internal desire comparing to other types of IT artifacts

3. This technique is a straightforward way to increase knowledge [6].

As a result, it is evident that Saudi women prefer to use simple text messages to increase their knowledge about STDs such as HPV. Hence, the IT artifact that could assist in increasing and maintaining task selfefficacy to educate Saudi women about HPV infections is simple text messages that designed and guided by the HPV facts transmission model. These messages, written in Arabic, include links to educational videos, as well as facts about HPV.

In this current research, the authors designed a flowchart, which named HPV Women's Awareness (HPVWA). The authors embedded this flowchart into a 2-way interactive SMS messaging system, as explained in the next two sections (section 3.2 and 3.3).

\subsection{HPV women's awareness (HPVWA) flowchart}

Guided by the HPV facts transmission model, the HPVWA flowchart was designed to increase selfefficacy so that a woman would want to receive more information, and then to maintain that level. To enhance self-efficacy, the first author sent a message to the participants (see section 3.3 for the sample description). The message was in Arabic and included some facts regarding HPV, which are the threat and consequence of not been educated, and a simple motivational statement. An example of a threat statement is, "Do you know that HPV is a type of infection that is transmitted by sexual intercourse? Having a sexual relationship with someone who has had many partners are the reasons for HPV infections." One example of a consequence statement is, "Do you know that if you educate yourself about HPV, you will reduce the chance of having this infection? You probably will not come down with cervical cancer." A motivational statement example is "Increasing your sexual health knowledge is important for your health, and you can do it without much effort."

To maintain self-efficacy, the first author kept sending some messages (in Arabic) that included some motivational sentences, such as "Your health is your priority, so you can educate yourself easily by reading the messages that we send." The messages contained some advice regarding when, where, and how women can learn about HPV. For example, to address when: "pick a time when you are usually free and do not have anything to do"; to address where: "explore these ideas in a place where you feel comfortable"; and to address how: "use your laptop, tablet, and/or smartphone and visit the following trusted website and read". Figure 4 is the flowchart of HPVWA to enhance and maintain women's self-efficacy.

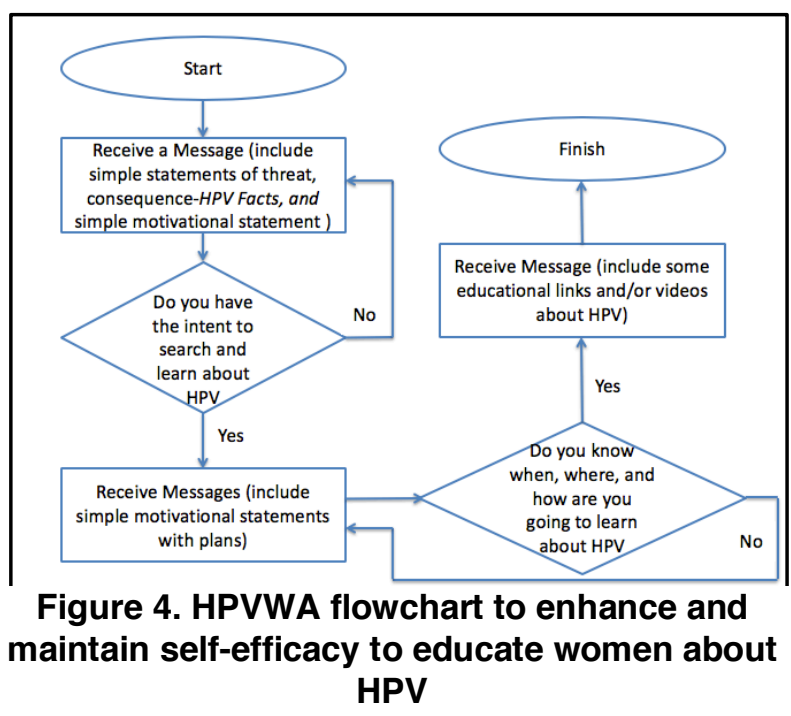

The authors wrote four different messages examples to enhance and maintain women's self-efficacy, two of which they used later as message contents during the study.

\subsection{The usage of the 2-way interactive messaging system, ExpertTexting (ET) platform with the HPVWA flowchart}


After the authors identified the HPVWA flowchart, they started to search for a platform that could be used to build the IT artifact or a ready-made platform that could be used to send the messages to the participants to increase and maintain their self-efficacy. The authors found a ready-made platform named ExpertTexting (ET) that is a 2-way interactive SMS messaging system, which supports international messages [18]. This platform is usually used in marketing to interact with customers with a high level of security [18]. The reasons for choosing this platform are that it is easy to use, supports the Arabic language, allows attaching links that include educational videos or reports, is secure, and supports sending international messages to an individual and a group.

These reasons match the requirements that were specified by the participants in stage 3 . As a result, the authors decided to use this platform instead of building an IT artifact from scratch. Also, there are several other features for this system. The ET platform has a dashboard where the authors can see all the messages that are successfully sent and the messages that failed, as shown in Figure 5.

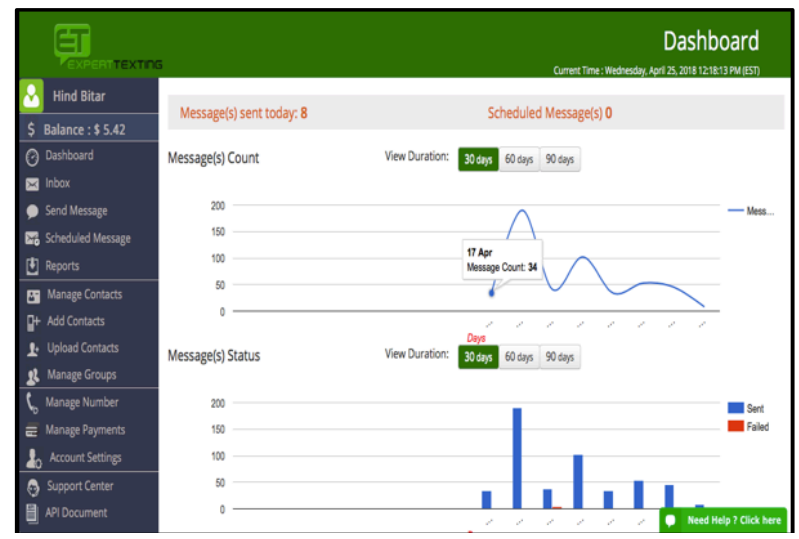

Figure 5. ET dashboard

Then, the first author started to recruit participants using convenience (especially snowball) sampling. Through this method, the authors contacted 34 Saudi women; all of the participants were living in Saudi Arabia and from the general public. The age of the participants was 20 and above because women who are diagnosed with cervical cancer are usually aged 20 and above with various degrees of frequency [12]. Each participant was identified with an ID number (e.g., P1, P2, P3) to avoid collecting personal information. The first author called each participant to get her verbal approval to participate in this study. At the same time, the goal, instructions, and steps of the study were clarified during the call, and each participant was informed of her ID and asked to use this ID to protect her privacy when sending messages.
Additionally, the participants were asked about their preferred day and time to receive the messages. All the participants indicated that they could receive the messages at any day or time, but they prefer the morning time. Subsequently, the first SMS message was sent using the ET system to welcome them. On the next day, the first author sent an Arabic version of the General Self-Efficacy (GSE) scale (Pre-GSE) [19], that developed using Google forms, to measure their selfefficacy before receiving the messages. The day after the participants filled out the survey, the first author sent a group of short messages to enhance their self-efficacy. The authors followed the HPVWA flowchart (see Figure 4) in sending the messages to the participants. The authors did not encounter any issues concerning increasing self-efficacy; from the first attempt, all the participants stated that they had the intent to search and learn about the stigmatized disease, HPV.

Then, the authors moved to the next phase to maintain their self-efficacy by sending a motivational statement and plan regarding where, when, and how they could search and learn; in the first attempt, eight of the participants stated that they did not have a detailed plan, while 26 women stated that they did. Therefore, the first author sent another motivational statement and detailed plan to maintain participants' self-efficacy for the eight of them who did not have a plan. Then, the first author asked them again: "do you know when, where, and how you are going to learn about HPV?" In that second attempt, all eight participants said yes, as shown in Figure 6.

Finally, the first author sent a link of the GSE scale to the participants to measure their self-efficacy after receiving the messages (Post-GSE). The length of time between sending the first (welcome) message and sending the Post-GSE was eight days.

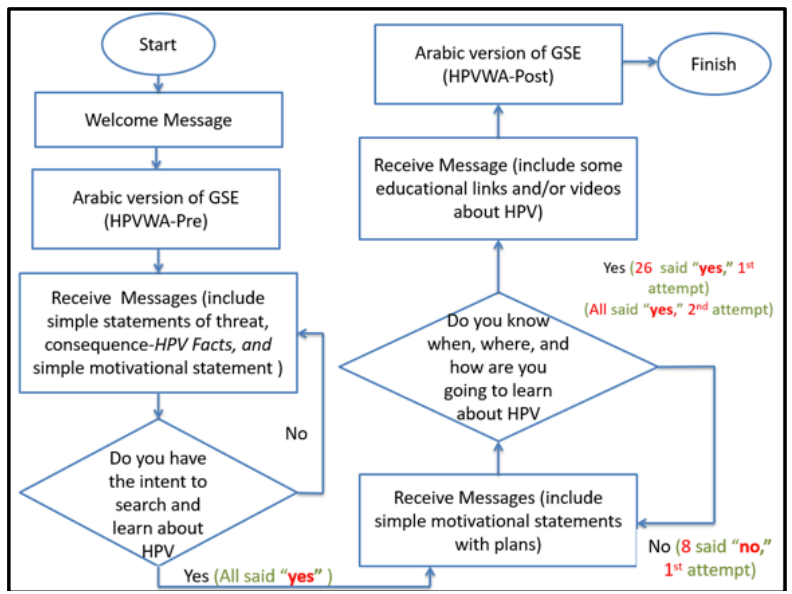

Figure 6. The usage of ET platform with the HPVWA flowchart, study duration eight days 


\section{Evaluating the IT solution}

The authors evaluated the effectiveness of using a ready-made, 2-way interactive SMS messaging system platform that applies the HPVWA flowchart to increase and maintain self-efficacy to educate Saudi women about HPV. To this purpose, we evaluated this technology from a socio-technical perspective by conducting a quasi-experiment, where the authors could identify women with high self-efficacy. According to Harris et al., quasi-experiments are defined as nonrandomized studies that aim to evaluate interventions [20]. The main goal of quasi-experiments is to validate causality between an intervention and an outcome [20]. The authors evaluated the use of a 2-way interactive SMS messaging system that applies the HPVWA flowchart using a one-group, pre-post intervention method. ${ }^{1}$ The authors recruited the subjects using convenience sampling to get access to some participants quickly. Then they used snowball sampling, asking each participant to suggest other possible participants. To see if there was a statistically significant difference in selfefficacy before and after using the 2-way interactive SMS messaging system with the HPVWA flowchart, the authors used the dependent samples t-test (paired samples t-test). The sample size $(\mathrm{n}=34)$ is larger than the minimum needed for a single group [21]. To determine the exact sample size, the authors used the "AI-Therapy Statistics" website, selecting the test family (t-test), sample groups (same subjects), number of tails (two), medium effect size (0.5), significance level $(\alpha)(0.05)$, and power $(0.8)$. This site advised that the sample size should be 34 .

There were 11 single women and 23 married women. The age range of single participants was from 20 to 42 , with a mean age of 26.5 , while the age range of married participants was from 22 to 56 , with a mean age of 32. All the participants had at least a B.A. Eighteen of the participants had a job. Participants lived in a variety of Saudi cities.

The authors analyzed the data focusing on the marital statuses (married, single), and the age categories (20-29, 30-39, and 40 and above). The evaluation stages are shown in Figure 7.

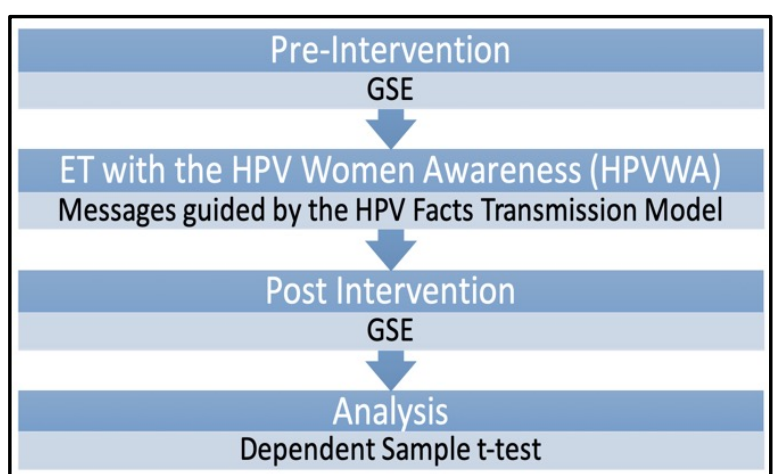

Figure 7. The evaluation stages of using the ET platform with the HPVWA flowchart

\section{Findings and discussion}

Before analyzing the data, the authors asked participants, "Did you watch and/or read the materials that we sent?" All the participants answered, "Yes."

First, the authors utilized descriptive statistics for the Pre-GSE and the Post-GSE scores, as shown in Table 1.

\section{Table 1. Participants' descriptive statistics}

\begin{tabular}{|c|c|c|c|}
\hline \multicolumn{4}{|c|}{ Participants' Descriptive Statistics } \\
\hline & & Pre_GSE & Post_GSE \\
\hline \multirow[t]{2}{*}{$\mathrm{N}$} & Valid & 34 & 34 \\
\hline & Missing & 0 & 0 \\
\hline \multicolumn{2}{|c|}{ Mean } & 29.41 & 33.79 \\
\hline \multicolumn{2}{|c|}{ Median } & 30.00 & 33.50 \\
\hline \multicolumn{2}{|c|}{ Mode } & 30 & $33^{\mathrm{a}}$ \\
\hline
\end{tabular}

The median for the Pre-GSE is 30.00 , and it is 33.50 for the Post-GSE. The median score is the most critical number to discover, since, according to Schwarzer and Jerusalem (1995), this score is the cut-off point that is used to indicate low or high self-efficacy of a group of research subjects [22].

The authors checked all the assumptions for the paired samples t-test since this is the analysis method that applied. The assumptions are (a) the dependent variable is continuous, (b) the observations are independent of one another, (c) the distribution of the dependent variable is normal, and (d) there are no outliers in the dependent variable [23]. All the assumptions were met in the data. The Pre-GSE and the Post-GSE scores are continuous. The observations are independent of one another since the occurrence of one observation provides no information about the occurrence of the other observation. The data is

\footnotetext{
${ }^{1}$ (Claremont Graduate University's IRB approved this study as expedited research [IRB \#3101].)
} 
normally distributed, and there are no outliers (skewness $=0.436$ ), as seen in Figure 8.

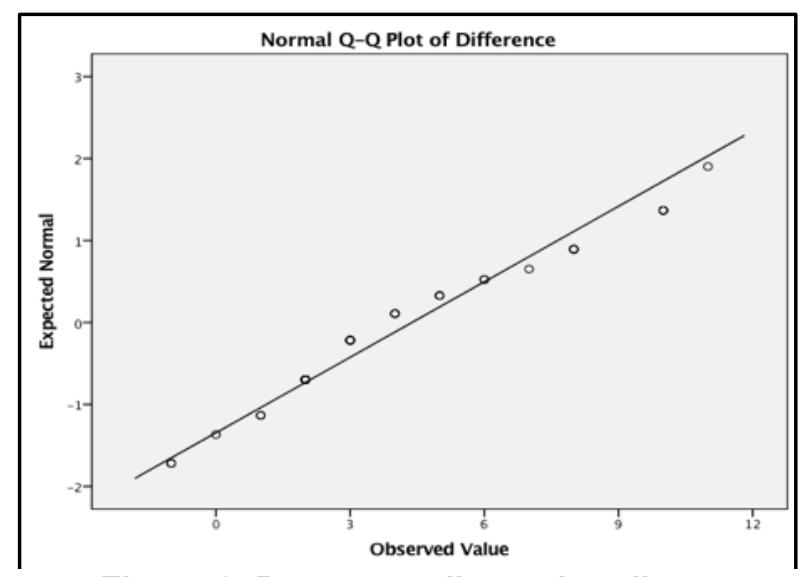

Figure 8. Data normality and outliers

After conducted the paired samples t-test for all the participants, the result showed 2-tailed significance at the 0.0001 level, which is lower than the specified pvalue of 0.05 . Therefore, there is a statistically significant difference in self-efficacy before and after using the 2-way interactive SMS messaging system with the HPVWA flowchart. There is evidence of causality between the IT artifact, using the ET and the HPVWA flowchart, and the outcome of the participants' selfefficacy.

To investigate the results for different categories, the authors analyzed the data again using the paired samples t-test but focused on the participants' ages to see if the results would be different. The authors divided the data into three groups based on the participants' age. The groups are (a) participants ages 20 to 29, (b) participants ages 30 to 39, and (c) participants ages 40 and above. The results show that there is no statistically significant difference in self-efficacy before and after using the 2way interactive SMS messaging system for just the group of women 40 and above. However, for the two other groups, the results are significant.

Fifteen participants were between 20 and 29 years old (inclusive), and another fifteen were between 30 to 39. The 2-tailed significance value for both groups is 0.0001 . This value is lower than 0.05 , so the results for these groups are significant. For the last group in this category, age 40 and above, the number of participants is four. The 2-tailed significance value is 0.066 , which is higher than 0.05 . This value indicates that there is no statistically significant difference in self-efficacy before and after using the messaging system. The reason for having non-significant results could be the small sample size.

Finally, the authors analyzed the data using a similar analysis method with a paired samples t-test, concentrating on two other categories, marital status (single $(\mathrm{n}=)$ ) 11, married $(\mathrm{n}=)$ 23) and cities' location (western $(n=) 22$, eastern $(n=) 7$, and central $(n=)$ 5). The results show that there is a statistically significant difference for these groups in self-efficacy before and after using the 2-way interactive SMS messaging system with the HPVWA flowchart. The 2-tailed significance value for marital status is 0.0001 . Similarly, the 2-tailed significance value for women who live in the western region is 0.0001 , the central region is 0.001 , and eastern region is 0.030 .

\section{Discussion and research implications}

The results of using a 2-way interactive SMS messaging system platform that applies the HPVWA flowchart to increase and maintain self-efficacy to educate Saudi women about HPV were statistically significant. This means that on average, after receiving the messages, Saudi women's self-efficacy increased and maintained; they believed in their ability to educate themselves about HPV. However, for Saudi women ages 40 and above, the results were not statistically significant. Consequently, receiving the messages did not assist in increasing and maintaining women's selfefficacy, so they did not believe in their ability to educate themselves about HPV. Nonetheless, for this group, it may be that a small sample size affected the result since there were just 4 participants in this group.

As stated by Lee et al. (2019), mobile-phone-based interventions could help spur behavioral change, but little is known about how these interventions could be successfully developed to prevent cervical cancer caused by HPV. The authors developed a tailored mobile text messaging intervention, mobile screening (mScreening), based on Korean-American immigrant women's cultural and individual needs. The mScreening interventions showed the effectiveness and feasibility of using mobile phones to increase women's knowledge regarding cervical cancer caused by HPV. In this study, all participants agreed that their mobile phone is an important part of their lives, so designing and developing a text messaging intervention could help address the lack of HPV knowledge, especially if it included video clips or images as well as facts about this disease. Additionally, some participants suggested designing an interactive messaging system that included some questions and answers to decrease some barriers of a one-way text messaging system [24].

Even though, there are several types of IT artifacts that may be used to enhance and maintain Saudi women's self-efficacy, such as a gamified web application, the decision of using this persuasive health technology is made based on what is previously learned from surveys of Saudi women. Saudi women preferred 
to learn alone about matters like HPV using a simple text messaging system, and this research supports this conclusion. Henceforth, the use of a 2-way interactive SMS messaging system could empower and equip Saudi women with facts and knowledge about HPV.

\section{Conclusion, contributions, limitations, and future research}

Technology utilization can assist in solving the problem of lack of transmission of HPV facts. Therefore, using a ready-made 2-way interactive SMS messaging system platform that applies the HPVWA flowchart-for the first time- was useful to increase and maintain women's self-efficacy to educate themselves about HPV in Saudi Arabia, which is the theory of the solution. Using this technology-assisted in reducing the effect of some barriers and resources factors of the "HPV Facts Transmission Model." For example, this system reduced the effect of the language barriers factor by providing some links and educational videos in the Arabic Language. Using the messaging system assisted Saudi women in learning HPV facts alone and secretly, which is crucial because of the stigma attached to the disease. Learning secretly with short messages decreased the negative impact of these barriers.

The main contribution to this research is to the health of women in society since using a messaging system assists Saudi women in increasing and maintaining their self-efficacy to educate themselves about HPV infections that cause cervical cancer. This system, ET, which is usually used in marketing and business, was used for the first time in the health sector to enhance and maintain Saudi women's self-efficacy. This artifact is a simple messaging system that was used to implement the HPVWA flowchart such that the study participants received regularly scheduled messages to enhance and maintain their self-efficacy. The design of a messaging system and the HPVWA flowchart comprise motivational statements, HPV Facts, and educational links and videos about HPV infections. Moreover, the technology informed some knowledge regarding when, where, and how women can learn about matters like HPV infections. Saudi women may not feel less informed about HPV infections if they did have this tool, so their self-efficacy scores would not be increased or maintained. Additionally, this artifact aided the Saudi women participants to learn secretly about some of HPV facts. This study also contributes to the body of IS research by adapting a two-way interactive SMS messaging system to increase and maintain Saudi women's self-efficacy to educate themselves about HPV in a way that meets their needs. Although this study provides a demonstration that IT can be used to alleviate the problem of lack of transmission of HPV facts, it has a few limitations.

One limitation of this research study is the sample size. Even though the authors used the "AI-Therapy Statistics" website to determine the sample size, the results could be different if we had a larger sample, especially for the group of women ages 40 and above.

To improve the evaluation design of the usage of the ET with the HPVWA flowchart, the researchers will apply a randomized controlled trial (RCT) for two groups (control and intervention). This makes it possible to see the effect of the messaging system has on women to enhance and maintain their self-efficacy. Furthermore, to educate Saudi women regarding HPV using this the ET SMS messaging system, the messages must include more facts about HPV prevalence in young adults, HPV types, high and low risks of HPV, how to diagnose it at an early stage, and ways to prevent this infection.

Lastly, educating women about STDs, such as HPV, is suggested to be strongly associated with women's quality of life. Women's health education is an essential concept that could help in reducing and preventing diseases and death around the world. Women's health could affect the wellbeing of their families and communities [25]. Their illness or death can negatively influence the health of their children, families, and communities. Increasing women's health knowledge plays a critical role in maintaining the wellbeing of their families and communities [25].

\section{References}

[1] L.P. Wong and I.C. Sam, "Ethnically diverse female university students' knowledge and attitudes toward human papillomavirus (HPV), HPV vaccination and cervical cancer," European Journal of Obstetrics \& Gynecology and Reproductive Biology, 148(1), 2010, pp. 90-95.

[2] J.M. Walboomers, M.V. Jacobs, M.M. Manos, F.X. Bosch, J.A. Kummer, K.V. Shah, P.J.F. Snijders, J. Peto, C.J.L.M. Meijer, and N. Muñoz, "Human papillomavirus is a necessary cause of invasive cervical cancer worldwide", The Journal of Pathology, 189(1), 1999, pp. 12-19.

[3] G.M. Clifford, J.S. Smith, M. Plummer, N. Munoz, and S. Franceschi, "Human papillomavirus types in invasive cervical cancer worldwide: a meta-analysis," British Journal of Cancer, 88(1), 2003, pp. 63-73.

[4] The World Health Organization, "Cervical cancer," https://www.who.int/cancer/prevention/diagnosisscreening/cervical-cancer/en/, published Sep 2018, retrieved May 2019.

[5] H. Bitar and T. Ryan, "Designing technology to overcome the lack of transmission of HPV facts: step one - a theory of 
the problem," Proceedings of the 51st Hawaii International Conference on System Sciences, 2018, January.

[6] H. Bitar, S. Alismail, and T. Ryan, "Understanding the lack of transmission of HPV facts and theorizing an IT solution to women in Saudi Arabia: a qualitative case study," Manuscript submitted for publication, 2019.

[7] M.L. Markus, "Maybe not the king, but an invaluable subordinate: a commentary on Avison and Malaurent's advocacy of "theory light" IS research," Journal of Information Technology, 2014, pp. 341-345.

[8] F.S. Alhamlan, M.N. AlAhdal, A.S. Al-Zahrani, and S.A. Almatrrouk, "Human papillomaviruses: The cervical cancer saga in developing countries," The Journal of Infection in Developing Countries, 11(11). 2017, pp.819-825.

[9] G. Nabi, "Knowledge of Saudi female university students regarding cervical cancer and acceptance of the human papilloma virus," Saudi Medical Journal, 36(2), 2015, pp. 254-255.

[10] F.S. Alhamlan, H.H. Khayat, S. Ramisetty-Mikler, T.A. Al-Muammar, A.M. Tulbah, I.A. Al-Badawi, W.I Kurdi, M.I. Tulbah, A.A. Alkhenizan, A.N. Hussain, and M.

Ahmed, "Sociodemographic characteristics and sexual behavior as risk factors for human papillomavirus infection in Saudi Arabia." International Journal of Infectious Diseases, 2016, pp.94-99.

[11] HPV Information Center, "Human Papillomavirus and related diseases report," https://hpvcentre.net/statistics/reports/SAU.pdf, retrieved August 2019.

[12] National Cervical Cancer Coalition, "Cervical cancer overview," $\quad$ http://www.nccc-online.org/hpvcervicalcancer/cervical-cancer-overview/, published Jun 2016, retrieved Jun 2019.

[13] G.K. Al-Shaikh, E.M. Almussaed, A.A. Fayed, F.H. Khan, S.B. Syed, T.N. Al-Tamimi, and H.N. Elmorshedy, "Knowledge of Saudi female university students regarding cervical cancer and acceptance of the human papilloma virus vaccine," Saudi Medical Journal, 35(10), 2014, pp. 12231230 .

[14] C.D. Walsh, A. Gera, M. Shah, A. Sharma, J.E. Powell, and S. Wilson, "Public knowledge and attitudes towards human papilloma virus (HPV) vaccination," BMC Public Health, 8(1), 2008.

[15] L.P. Wong, "Knowledge and attitudes about HPV infection, HPV vaccination, and cervical cancer among rural southeast Asian women," International Journal of Behavioral Medicine, 18(2), 2011, pp. 105-111.
[16] S.M. Shah, C. Ayash, N.A. Pharaon, and F.M. Gany, "Arab American immigrants in New York: health care and cancer knowledge, attitudes, and beliefs," Journal of Immigrant and Minority Health, 10(5), 2008, pp. 429-436.

[17] F.F. Sniehotta, U. Scholz, and R. Schwarzer, "Action plans and coping plans for physical exercise: A longitudinal intervention study in cardiac rehabilitation," British Journal of Health Psychology, 11(1), 2006, pp. 23-37.

[18] ExpertTexting, https://www.experttexting.com, published Jan 2018, retrieved Jun 2019.

[19] Academia, "Self-Competency Scale -Structure and Measurement", https://www.academia.edu/9372517/SelfCompetency Scale -Structure and Measurement, retrieved January, $201 \overline{8}$.

[20] A.D. Harris, J.C. McGregor, E.N. Perencevich, J.P. Furuno, J. Zhu, D.E. Peterson, and J. Finkelstein, "The use and interpretation of quasi-experimental studies in medical informatics", Journal of the American Medical Informatics Association, 13(1), 2006, pp. 16-23.

[21] Robson, C., and K. McCartan, Real World Research. John Wiley and Sons, 2011.

[22] R. Schwarzer and M. Jerusalem, "Generalized selfefficacy scale, in J. Weinman, S. Wright, and M. Johnston (Eds.), Measures in Health Psychology: A User's Portfolio, 1995.

[23] StatisticsSolutions, "Paired sample t-test," http://www.statisticssolutions.com/manova-analysis-pairedsample-t-test/, published 2018, retrieved Jun 2019.

[24] Lee, H. Y., Lee, M. H., Sharratt, M., Lee, S., \& Blaes, A. (2019). Development of a Mobile Health Intervention to Promote Papanicolaou Tests and Human Papillomavirus Vaccination in an Underserved Immigrant Population: A Culturally Targeted and Individually Tailored Text Messaging Approach. JMIR mHealth and uHealth, 7(6), e13256.

[25] Kerala Women, "The importance of women's health," http://www.keralawomen.gov.in/index.php/health/190-theimportance-of-women-s-health, published Sep 2012, retrieved Jun 2019. 